\title{
«La création de rentes : une approche par les compétences et capacités dynamiques »
}

\author{
$\underline{\text { Auteurs }}$ \\ Thierry Burger-Helmchen, Laurence Frank \\ Document de Travail n²011 - 01
}

Janvier 2011

Faculté des sciences économiques et de gestion

Pôle européen de gestion et d'économie (PEGE)

61 avenue de la Forêt Noire

F-67085 Strasbourg Cedex

Secétariat du BETA Géraldine Manderscheidt Tél. : (33) 0368852069 Fax : (33) 0368852070 g.manderscheidt@unistra.fr http://cournot2.u-strasbg.fr/beta 


\section{LA CREATION DE RENTES : \\ UNE APPROCHE PAR LES COMPETENCES ET CAPACITES DYNAMIQUES}

Thierry Burger-Helmchen

BETA, EM Strasbourg, Université de Strasbourg burger@unistra.fr

Laurence Franck

BETA, EM Strasbourg, Université de Strasbourg

lfrank@cournot.u-strasbg.fr 


\section{LA CREATION DE RENTES : UNE APPROCHE PAR LES COMPETENCES ET CAPACITES DYNAMIQUES}

\section{I. - INTRODUCTION}

Ces dernières années la théorie de la firme s'est principalement développée suivant deux axes, (i) celui de la prise en compte des actifs intangibles, notamment la connaissance et les routines, et (ii) la prise en compte de la dynamique de développement de la firme au lieu d'une vision de statique comparative.

(i) La prise en compte des actifs intangibles comme étant les sources de valeur les plus importantes de la firme a permis d'apporter des réponses différentes aux questions fondamentales posées à chaque théorie de la firme (existence, frontière, organisation) et de réorienter la problématique de la répartition des ressources de l'économie vers la production (Azoulay et Weinstein, 2000).

(ii) Une vision dynamique de la firme a permis de mettre en évidence les interactions entre les éléments de la firme et son environnement. Il appartient à la firme de pouvoir s'adapter, d'anticiper ou d'initier les changements de l'environnement afin de s'approprier durablement un gain dans un cadre dynamique (Hodgson, 1998 ; Porter, 1991; Rathe et Witt, 2001).

Dans ces deux approches l'objectif de la firme est de modifier sa capacité de production afin de mettre sur le marché des produits et des services rentables. Or, dans un cadre dynamique ces rentes ne sont généralement pas durables, aussi des formulations théoriques se sont développées pour distinguer les caractéristiques des firmes et des ressources capables de créer et de conserver des rentes sur une durée conséquente. Les capacités dynamiques (Teece et al., 1997 ; Dosi et al., 2000) correspondent à une telle formulation répondant au besoin d'intégrer les changements internes et externes que subit la firme. Mais derrière ce label se cachent des réalités diverses où le lien entre la spécificité des actifs et celle des rentes qu'ils sont sensés générés sont parfois tronqués.

Ce travail passe précisément en revue les principales contributions au concept de capacités dynamiques et de rentes afin de définir les liens qu'entretiennent ces deux notions. Ceci conduit à l'élaboration d'un mécanisme de choix d'options d'évolution futur pour la firme. En effet s'il est reconnu que les ressources de la firmes sont à l'origine des rentes et que les capacités dynamiques déterminent la portée de ces rentes peu d'indications sont offertes quant à la nature de la rente à rechercher et du choix des actifs à développer. Notre proposition et que ce choix peut être analysé comme une décision de création et d'exploitation d'options.

Le graphique 1 décrit la situation dans laquelle nous nous plaçons dans cet article. Sur ce graphique sont représentées des courbes d'isoprofits (I et II) et trois firmes (A, B, C) qui ont fait des choix de développements différents. Un déplacement vers la droite de la courbe d'isoprofit correspond à une augmentation du niveau de profit. Les firmes $\mathrm{A}$ et $\mathrm{B}$ ont un même niveau de profit mais leurs arbitrages en terme de coûts de production et de variété sont différents (l'hypothèse généralement admise que l'augmentation de la variété et de la flexibilité s'accompagne d'un accroissement plus que proportionnel du coût est faite ici). La firme A a choisit de produire une plus grande variété de biens 
tandis que la firme $\mathrm{B}$ a choisit de se spécialiser. La firme $\mathrm{C}$ quand à elle est à un niveau de développement de ses connaissances plus avancé qui lui permet à la fois de produire une plus grande variété de bien à un coût peu élevé.

Le passage du point $\mathrm{A}$ au point $\mathrm{C}$ peut se faire en suivant différent sentiers (graphique 1.a). Soit la firme reste sur un même niveau de coût mais arrive à augmenter la variété des biens quelle produit (flèche 1), soit elle choisit une stratégie basée sur une réduction préalable des coûts associée à une baise de variété pour ensuite augmenté à nouveau la variété. Ce qui correspondrait à se rencontrer sur ses fondamentaux pour ensuite se développer vers une autre direction (flèche 2 puis 3). La firme au point B se trouve devant un choix symétrique. Le graphique 1.b représente l'ensemble des options qui s'offre à chaque firme parmi lesquelles elle peut choisir son évolution futur. On peut remarquer que certaines de ces options conduisent les firmes à un même emplacement sur la courbe d'isoprofit (équifinalité des choix).

\section{Insérer ici Figure 1}

D'une part ces options sont contraintes par les ressources que détient la firme et sa son habileté à les employer dans de nouvelles activités. D'autre part ces options spécifient le type de rente que la firme peut obtenir. Or, si certaines firmes peuvent plus facilement se développer suivant une dimension (coût ou variété) certaines rentes devraient être plus faciles à obtenir que d'autres et par là contraindre à nouveau le choix d'options futurs.

Ce mécanisme de choix est fortement dépendant des définitions de capacités dynamiques et de rentes employées. Si l'acquisition d'une rente quelconque, que nous considérons ici comme l'objectif de la firme, provient d'une combinaison d'actifs tous les actifs d'une firme ne peuvent pas être la source d'une rente. Les rentes sont générées exclusivement par une catégorie d'actif labellisée « ressources » (Barney, 1991 ; Peteraf, 1993). Les ressources se distinguent des facteurs de production sur quatre points: une ressource est rare, à une valeur dérivée de son un emploi dans le processus de production de la firme, est inimitable et ne peut se faire substituer par un autre actif. Si ces quatre conditions sont réunies la ressource permet à la firme de se constituer un avantage durable (sustainable advantage). Pour Barney (1991:102) un avantage est durable lorsqu'une firme met en place une stratégie qu'aucune autre firme ne peut imiter à court terme. Une firme a un avantage compétitif non seulement vis-à-vis de tous ses concurrents sur le marché, mais également de tous ses concurrents potentiels, d'où l'importance accordée à l'environnement de la firme et des capacités de réaction voir de proaction des firmes concurrentes introduites dans la théorie des capacités dynamiques.

Afin de clarifier le vocabulaire et de distinguer de façon pertinente parmi les notions employées la suite de ce travail sera consacrée tout d'abord à une revue de la littérature des concepts de compétences et de capacités dynamiques qui cherchent à caractériser l'évolution de la firme par rapport à ses ressources et à son environnement. Un travail similaire est ensuite mené sur les nombreuses définitions de rente existantes. Ces démarches ont pour but de mettre en exergue les caractéristiques communes entre les capacités dynamiques et les rentes et leurs interactions. Le lien entre ses deux notions nous conduit alors à proposer un modèle conceptuel de choix d'options à developper. 


\section{II. - Compétences et capacités dynamiques}

Si la connaissance et plus généralement les ressources sont à l'origine des rentes de la firme, celles-ci n'ont pas vocation à durer dans un environnement en mutation où les firmes doivent suivre si ce n'est induire la dynamique du changement. Les capacités dynamiques correspondent à une formulation théorique répondant au besoin d'intégrer les changements que subit la firme. Mais derrière ce label se cachent des réalités diverses.

\section{II.1. Définition}

Le concept de compétences/capacités dynamiques, sous une dénomination ou une autre, correspond à une idée déjà ancienne dans l'approche de la firme. Ainsi, on peut interpréter la notion de "distinctive competence » de Selznick (1957) comme une des premières formulations de ces capacités dont le but est de transformer la firme en une entité plus adaptée à son environnement actuel et futur. La définition de Selznick est des plus générales « The transformation of an engineered, technical arrangement of building blocks into a social organism », les développements ultérieurs détailleront davantage le lieu, les éléments, et les moyens de cette transformation.

Le tableau suivant reprend les principales définitions données dans la littérature sur ce que sont les compétences dynamiques:

\section{Insérer ici Tableau 1}

De ces définitions on peut dire que les compétences dynamiques sont le résultat (i) d'une somme de processus ou de routines (ii) dont le but intentionnel est d'adapter la firme à l'environnement et de lui permettre d'exploiter des opportunités.

De ce fait, les capacités dynamiques rejoignent sur de nombreux points de définitions les objectifs de la stratégie au sens de Porter (1994). La stratégie correspond au développement de compétences différenciées afin d'exploiter au mieux des opportunités. Cependant la stratégie est développée à un seuil décisionnaire de la firme, tandis que les capacités dynamiques représentent des décisions et des actions à plusieurs niveaux de la firme. Il faut donc s'attendre à ce que les capacités dynamiques soient un ensemble de processus, chacun exerçant une activité à un niveau différent de la firme. L'effet combiné de ces processus conduit à un changement majeur dans la position de la firme.

L'anarchie dans les définitions existantes des capacités dynamiques connaît deux sources principales. Outre (i) un problème récurent de disparités dans les définitions, on note (ii) des dissimilitudes dans le niveau d'analyse retenu (l'organisation, la firme) et la nature des éléments sur lesquels ces compétences s'exercent (intangible, tangible).

(i) La relative confusion évoquée porte sur les concepts de «capabilities » et de « capacities » que les traductions en français n'améliorent pas. Les « capabilities » synonyme de compétences et savoir-faire nécessaires à créer et guider le changement dans une firme se trouvent au début du processus de changement (Azoulay et Weinstein, 2000; Foss, 1996). Les « capacities » correspondent à la 
capacité productive de la firme et sont le résultat du changement comme par exemple la modification de la fonction de production de la firme. L'approche que nous retenons dans la suite, et qui nous paraît la plus simple est de parler de «capacités dynamiques" lorsque capacités fait référence à "être capable de produire » un certain type de bien, dans certaines conditions (quantité, qualité, coût, prix...) et de réserver le mot compétence dynamiques aux éléments intangibles correspondant aux « capabilities » anglais.

(ii) Les capacités dynamiques s'expriment par les modifications des positions de la firme, en terme de facteurs de production, ressources, compétences et outputs produits. Ces positions ont une forte dépendance de sentier et conditionnent les trajectoires futures. Si les capacités dynamiques s'appliquent à la firme dans son ensemble, les compétences dynamiques quant à elles ne regroupent que des actifs intangibles. Par définition une capacité dynamique n'est pas un effet à un moment donné mais un processus qui s'accompli sur une durée. Teece et al.(1997) avancent l'idée que les compétences de la firme sont la résultante de plusieurs processus, chaque processus étant composé de connaissances et de savoir-faire individuels et d'une routine particulière qui lient les individus aux cours du processus. Le nombre de processus dépend du niveau d'analyse retenu. On peut par exemple répertorier les processus de production, de coordination, de décision l'ensemble est chaperonné par un processus organisationnel ou si l'accent est mis sur les individus, on parlera de processus entrepreneurial ou managérial.

Il semble que Teece et al.(1997) et Kogut et Zander (1992) soient les seuls à souligner explicitement les liens entres actifs tangibles et intangibles dans l'obtention de capacités dynamiques. Un lien entre compétences et capacités est proposé dans le point suivant.

\section{II.2. Une hiérarchie des compétences et des capacités dynamiques}

Une compétence dynamique n'a d'utilité que dans un environnement dynamique. Dans un cadre statique, la firme n'a pas d'intérêt à subir un coût pour être en mesure de changer suite à une modification de l'environnement si ce dernier ne change jamais. Il convient de différencier les changements en réaction à un choc exogène des changement induits par la firme.

En absence d'un choc extérieur, la firme peut induire le changement et modifier sa capacité de production. Winter (2000) propose un classement par niveau des compétences dynamiques. Ce classement lui permet de différencier les compétences dites dynamiques des routines organisationnelles. Cette différenciation se fait suivant le consensus établi dans la littérature, que les compétences dynamiques sont à l'origine d'un changement en opposition aux routines organisationnelles qui sont axées sur la mise en place et l'amélioration du changement. On remarque ici que non seulement les compétences doivent évoluer et mais aussi se diffuser (et sont donc susceptible d'intervenir à plusieurs niveaux) afin d'investir la firme dans son ensemble et transformer les capacités de production. La capacité de production étant la résultante des compétences dynamiques elle est nécessairement d'un niveau inférieur ou égal à celui des compétences dynamiques. L'approche que l'on suggère pour caractériser la diversité des capacités dynamiques reprend l'argumentation stock-flux de Dierickx et 
Cool (1989). Si la capacité de production d'une firme est le résultat d'une accumulation d'actifs qui vont rester plus ou moins durablement au sein de la firme les compétences dynamiques vont modifier cette capacité par des flux d'actifs.

\section{Le patrimoine minimal de compétences et de capacités de la firme}

Winter (2000) dans une analyse des capacités dynamique retient que le niveau « $O$ » est celui qui permet à la firme de survivre face à un choc exogène. Il n'y a pas d'innovation selon la classification de Schumpeter. Il s'agit de compétence dans la mesure où ce choc peut influencer la fonction de production via les inputs disponibles pour la firme. Cette dernière doit alors trouver un substitut viable. Comme il est peu probable que la firme puisse avoir un substitut parfait, sa frontière de production et implicitement ses connaissances vont devoir se modifier de façon à obtenir une nouvelle frontière de production.

Au niveau organisationnel, ces compétences impliquent que la firme peut produire de la façon la plus efficiente, donc se situer sur le frontière de production. Elle est en mesure de circonscrire tout comportement opportuniste des agents qui l'empêche de se positionner sur la frontière de production. Ces compétences de niveau 0 dote la firme d'un mécanisme que Winter (2003) qualifie de « ad hoc problem solving » qui lui permet, suite à un choc exogène, de ne pas avoir un comportement erratique. Ils s'agit de comportements qui ne sont pas répétitifs, qui sont rationnels au moins dans l'intention, qui ne correspondent pas à des routines et ne sont pas conditionnés. C'est une réponse à une nouvelle situation, exogène et non prévue par la firme. Il y a des éléments de routines dans les comportements $a d$ hoc dues à l'éducation, l'expérience etc.

Les compétences dynamiques incorporent certainement des réactions ad hoc, aussi la frontière entre les compétences dynamiques (supérieur au niveau 0 ) et ce niveau de base n'est pas une frontière séparatrice mais gigogne. Sans ces compétences de niveau 0 la firme ne serait pas en mesure de transformer les inputs en outputs et d'en tirer un profit d'une façon efficace et durable.

\section{Les niveaux 1 à $N$}

Si les compétences modifient le processus de production ou créent de nouveaux produits les compétences sont d'un niveau supérieur (sous-entendu supérieur à 0). Une compétence de ce niveau implique que la firme peut modifier une dimension de sa fonction de production. Elle est en mesure d'apprendre et de rechercher des connaissances afin de déplacer sa fonction de production vers un nouveau maximum selon la dimension impliquée. Au niveau organisationnel ce type de compétences se manifeste par l'intégration des nouvelles connaissances et de la faculté à produire au plus près de la fonction de production théorique.

Une capacité de niveau $N$ correspond à la modification de la frontière de production suivant $N$ dimensions. Si $N$ est élevé la firme produit un changement majeur, comme lancer un produit entièrement nouveau voire créer une nouvelle industrie.

Les compétences dynamiques peuvent s'exprimer à plusieurs niveau via les processus et donc modifier un nombre de dimensions variables suivant la firme considérée. Par exemple un franchiseur a des capacités pour créer et produire de nouveaux biens mais également des compétences pour implanter des nouveaux magasins franchisés. Si cette 
activité de recherche de nouvelles localisations est inhabituelle alors la mener a bien nécessite des compétences de niveau supérieur qui vont agir sur plusieurs dimensions de la firme car elles impulsent un changement dans l'activité principale de la firme. La complexité de la typologie dépendant de la finesse de l'analyse souhaitée (du nombre de dimensions retenues).

Dans cette approche que devient la critique formulée, entre autre, par Collis (1994). Pour cet auteur, l'approche des compétences organisationnelles conduit à une rhétorique infinie sur l'apprentissage où le plus important pour la firme est d'apprendre à apprendre à apprendre... Dans le référentiel présenté cette régression infinie est bornée par le nombre de dimensions. Si l'une de ces dimensions représente la R\&D dont l'activité est de produire de la nouvelle connaissance, alors la compétence dynamique associée correspond à améliorer la R\&D, donc apprendre à apprendre. L'avantage de cette approche est de prendre la compétence associée comme le niveau le plus élevé et de porter l'analyse sur les processus que l'on désire retenir.

\section{Comment guider l'évolution? Quels éléments intégrer?}

La firme, via ses compétences dynamiques, va s'attacher les services de certaines ressources pour une longue période. Les choix des dimensions à développer entraîne des irréversibilités. Aussi, les capacités dynamiques sont une approche de long terme, en contraste avec l'adaptation ad hoc qui s'apparente à une réaction de très court terme. Ainsi, plus le nombre de dimensions concernées est grand, plus le changement sera profond, durable et coûteux. A vouloir trop changer la firme s'impose des coûts supplémentaires. Ce changement n'est souhaitable que si la valeur compétitive de la nouvelle capacité surpasse l'ancienne compte tenu des coûts de changement.

Winter (2000) suggère des règles de satisfaction pour guider le choix des compétences à actionner au sein de la firme. Pour cet auteur la firme doit avoir un processus d'estimation ou d'appréciation des situations qui se termine lorsqu'une alternative satisfaisante a été découverte. Ces estimations précèdent l'action. Pour lui le processus de recherche explore un ensemble d'alternatives préexistantes, ces alternatives sont le plus souvent découvertes, plutôt que créées. Les critères de définition d'une alternative satisfaisante sont statiques. Mais ces hypothèses peuvent ne pas être vérifiées dans un cadre dynamique où par définition les niveaux d'aspirations vont évoluer.

Pour obtenir une nouvelle capacité de production, une firme doit faire une série d'investissements complémentaires et spécifiques dans des actifs tangibles et intangibles. La mise en place de cette nouvelle capacité influence les frontières de la firme. Un apprentissage significatif, en lien avec d'autres investissements, se produit alors. Cet apprentissage est le plus fort au niveau du développement d'un nouveau processus. Le processus de développement devient naturellement le point focal puisque c'est de sa réussite que va dépendre la réalisation des prochains investissements. Stankiewicz (2003) montre que les compétences dynamiques de la firme dépendent des choix organisationnels mais aussi des investissements stratégiques en matière de ressources humaines.

Les capacités dynamiques sont donc dépendantes de la firme et de ses processus mais également de l'environnement. Cette position est celle de Eisenhardt et Martin (2000) qui font une différenciation entre les marchés faiblement et fortement dynamiques. Pour 
ces auteurs un marché faiblement dynamique est caractérisé par une structure de l'industrie stable. Les frontières de la firme sont clairement définies ainsi que les business model qui guident les actions de la firme. Le changement dans cet environnement est relativement linéaire et prévisible. En opposition un marché dynamique a une structure ambiguë, les frontières de la firme et de l'industrie sont floues, de fréquentes entrées et sorties des acteurs donnent lieu à des changements imprévisibles. Aussi dans un marché dynamique les routines en place ne peuvent pas être aussi détaillées, mais reposent d'avantage sur des exécutions expérimentales que sur une connaissance spécifique de la situation. Ainsi, dans un marché dynamique la nature des capacités dynamiques est différente, les mêmes processus ne sont pas sollicités.

Si les compétences dynamiques, via les processus, s'appliquent à différents niveaux, cela est dû tout d'abord à la vaste palette d'actions que la firme peut entreprendre. C'est pourquoi Eisenhardt et Martin (2000) considèrent les capacités dynamiques comme un phénomène à la fois stratégique et organisationnel, donnant lieu au développement de produits nouveaux, mais également la création d'alliances entre plusieurs firmes.

Inclure des opérations de type dans la portée des compétences dynamiques signifie qu'elles ont une activité de longue durée et non pas un effet instantané en réaction à un choc. Une compétence dynamique n'est pas une compétence instantanée, mais une action qui s'exprime sur une durée.

Les capacités et les compétences ont des points communs entre les firmes. Il s'agit là de la recherche d'une meilleure pratique. Cependant, cette analyse que font Eisenhardt et Martin (2000) est uniquement valable dans le cas d'une réaction ad hoc ou d'une stratégie d'imitation. En effet, comme le montre Porter (1996) en parlant de l'imitation d'une meilleure pratique, si toutes les firmes opèrent selon cette meilleure pratique il n'y aura pas de gain hors normes et les firmes vont être homogènes sur un nombre de plus en plus grand de leurs éléments. Les compétences dynamiques peuvent avoir une finalité identique mais la façon dont elles vont atteindre le but fixé peut varier.

La co-évolution de la firme et du marché rejoint l'agenda de recherche de Rathe et Witt (2001) et permet de différencier les routines organisationnelles et les compétences dynamiques. La conception des routines qui sont une suite d'actions complexes et détaillées dans l'ensemble mais dont l'exécution est linéaire. Cette exécution linéaire n'est possible que dans le cas de compétences dynamiques et non pas de réaction ad hoc.

L'hétérogénéité des capacités dynamiques dépend de l'hétérogénéité des compétences dynamiques qui vont influencer le timing des investissements au sein de la firme. La valeur des capacités dynamiques dépend de la fonction de production de la firme obtenue qui permet de créer des flux de rentes spécifiques à actualiser. Même si les compétences dynamiques sont hétérogènes et spécifiques à la firme, leurs effets présentent des points communs d'où une similitude dans les positions des firmes.

On s'éloigne de la conception classique des capacités dynamiques, faites de routines pour apprendre des routines, spécifiques à la firme, idyosincratiques, dont l'avantage provient des ressources qu'elles créent. La vision qui domine de plus en plus est celle de processus spécifiques grâce auxquels la firme transforme sa base de ressources. Ces processus ont des points communs d'une firme à l'autre (meilleure pratique). L'avantage 
n'est pas la ressource créée mais la capacité dynamique elle même, Eisenhardt et Martin (2000).

\section{II.3. L'origine}

La construction de nouvelles capacités productives est expliquée par Kogut et Zander (1992) par la recombinaison de compétences et connaissances existantes. Ils soulignent l'importance des liens sociaux entre les individus d'une même firme. Zollo et Winter (2002) décrivent les compétences dynamiques comme le chaînon entre les mécanismes d'apprentissage et l'évolution des routines opérationnelles. Ils classent les mécanismes d'apprentissage de la firme en trois groupes (i) Accumulation de l'expérience, (ii) Articulation de la connaissance (iii) Codification de la connaissance.

\section{Une évolution cyclique de la connaissance organisationnelle}

Zollo et Winter font un lien entre variation - sélection - rétention et l'évolution des connaissances organisationnelles. La variation peut avoir deux origines pour ces auteurs, externe ou interne. Un stimuli externe est une opportunité nouvelle créée par l'environnement et qui engendre un entrepreneur. Le mécanisme peut fonctionner de façon endogène, sans nouvelle stimulation de l'intérieur, dans ce cas les compétences proviennent de la co-évolution de plusieurs processus tacites et d'activités d'articulation de la connaissances et de codifications. La co-évolution repose sur la prise en compte simultanée de trois mécanismes d'investissement (i) financier, (ii) temporel et (iii) cognitifs.

L'investissement (pour développer les compétences dynamiques) est faible si la firme se base sur un processus d'accumulation de l'expérience. Dans ce cas la condition est une stabilité du personnel et éventuellement le recours momentanée à des expertsconsultants pour des tâches très spécifiques. Les coûts vont grandir si la tâche nécessite de rendre explicite une connaissance, en quel cas, le coût est surtout des coûts d'opportunités des individus. Llerena et Oltra (2002) présentent un travail de simulation sur les processus d'apprentissage interne et externe. La coexistence des processus d'apprentissage interne et externe conduit à long terme à une plus grande capacité des firmes à innover et exploiter les opportunités technologiques.

Des travaux de Zollo et Winter (2002) se dégagent quatre principes pour guider la genèse et l'évolution des capacités dynamiques : (i) La codification sert au transfert du know why et du know how. La codification permet d'exposer les liens entre les diverses actions et le niveau de performance. (ii) La codification doit avoir lieu à un moment optimal. Trop tôt elle conduit à généraliser à partir de trop peu d'expériences. Trop tardivement revient à codifier quelque chose qui a déjà été accepté soit par superstition soit par rationalité. (iii) La codification doit être soumise à l'adhésion des individus. (iv) Il faut une structure de support pour déterminer quels matériaux codifiés doivent être transmis à tous ou à des départements ou individus précis.

Ces travaux n'expliquent que partiellement l'origine des capacités dynamiques, il manque un objectif qui poussent à la création et au développement de telles capacités. L'obtention de rentes est une justification maintes fois avancée par les auteurs du domaine. Cependant les définitions de rentes sont multiples et leurs études permettent 
de préciser la nature des capacités dynamiques. C'est pourquoi le prochain point est consacré à un survol de cette notion aux interprétations multiples.

\section{III. - Rentes}

Pour Alchian (1988) et Muth (1968) il existe au moins trois significations différentes du terme rente dans la littérature: (i) L'usage commun, la rente synonyme de loyer. La rente est le prix de location que doit verser à intervalles réguliers l'usager d'un bien à son détenteur. (ii) L'usage en économie " classique ", la rente est un payement qualitativement différencié des salaires qui rémunèrent le travail et des intérêts qui rémunèrent le capital financier. La rente quant à elle rémunère la terre. (iii) L'usage dans la théorie économique de la firme où la rente correspond à la rémunération d'un input aux propriétés particulières. La rente se distingue donc du profit d'un point de vue qualitatif quant à la nature de l'input qui génère cette rente.

Cette troisième signification, qui comporte de nombreuses sous-catégories, est développée dans la suite de cette section. On aborde notamment les conditions qui définissent l'existence et le type de rente économiques. La pluralité des définitions, parfois contradictoires, amène une prolifération des classements des différents concepts de rentes. L'approche retenu ici est la même que pour les compétences dynamiques, on présente d'abord les rentes qui découlent d'un patrimoine déjà existant (un stock) puis celles qui proviennent d'une accumulation continue (flux) et enfin celles provenant d'une variation en majeure partie non anticipée.

\section{III.1. Les rentes à la Ricardo-Marshall}

L'idée de rente économique a été développée par Ricardo (1821) dans son argumentation pour l'abolition des « england's corn laws ». Ricardo fait l'observation que la terre est hétérogène du point de vue de la fertilité de telle façon que si la demande de grain devenait suffisamment forte pour que l'on se mette à exploiter les terres peu fertiles, les propriétaires des terres très fertiles (et donc rares) percevraient un supplément de revenu. C'est l'excès de la demande et la pénurie de terre qui crée un prix élevé et donc une rente élevée aux propriétaires terriens.

De cette approche, deux caractéristiques des rentes se dégagent: (i) Tout d'abord la rente provient de l'offre limitée d'un stock de ressource (la terre). La rente est donc une mesure agrégée du degré de rareté et d'utilité d'une ressource. Il emploie d'ailleurs la terminologie de "scarcity rents ». (ii) Ensuite Ricardo introduit la possibilité que sur un même marché cohabitent et subsistent des offreurs de ressources qualitativement différenciées. Puisque la particularité de la ressource qui crée la rente est d'être en offre fixe, une augmentation du prix ne peut pas attirer de nouvelles ressources dans cette utilisation, seul des ressources imparfaitement substituables (ici la terre de mauvaise qualité).

Cette approche permet de définir une mesure de la rente ricardienne, qui est une mesure relative à la terre la moins fertile employée. Cette mesure est reprise plus tard par des économistes qui font migrer le concept de rente de Ricardo de l'exploitation agricole aux firmes industrielles. On l'oppose à la mesure de Pareto qui est la différence 
entre le paiement de la ressource dans sa meilleure utilisation et le paiement dans sa seconde meilleure utilisation (ou son utilisation actuelle). Si les ressources utilisées sont parfaitement homogènes alors elles ont toutes la même meilleure utilisation alternative, et la même valeur, dans ce cas la rente de Ricardo est égale à la rente de Pareto. L'hypothèse d'homogénéité des ressources, difficile à concevoir avec des capitaux physique, ne tient évidemment plus lorsque l'on considère la firme du point de vue des connaissances. Le concept de rente a donc évolué pour s'intégrer de plus en plus dans la théorie de la firme.

\section{Les rentes dans la théorie de la firme, le développement de Marshall}

Marshall (1920) a défendu et développé l'approche de Ricardo. Pour Marshall la rareté que Ricardo accorde à la terre s'applique aussi aux autres inputs de la firme (ressources) qui existent en quantité temporairement ou définitivement fixe. Le développement fait par Marshall correspond en majeure partie à l'emploi du concept de rente fait dans la théorie de la firme basée sur les ressources. Il emploie les termes de « rente » et « quasirente» pour distinguer une ressource dont l'offre est définitivement fixe d'une ressource dont l'offre n'est que temporairement fixe. On retrouve au sein de cette approche de la rente "Ricardo - Marshalienne » appliquée à la firme les mêmes caractéristiques mises en avant uniquement pour la terre: (i) La rente est un phénomène qui s'applique exclusivement à un facteur dont l'offre est fixe ou quasi-fixe. (ii) La rente est un surplus, un excès de revenu par rapport à un benchmark qui correspond à la situation normale.

On comprend que les facteurs de productions triviaux, qui ne rassemblent pas toutes les caractéristiques énoncées par Barney (1991), comme les biens publics, ne permettent pas de générer une rente. Enfin, le fait qu'un actif génère une rente ne sous-entend pas que l'utilisateur de cette ressource perçoit la rente, il faut donc avoir un droit de propriété, ou du moins la capacité de s'approprier la rente générée par la ressource, Klein et al.(1978).

Ce concept de rente, comme l'illustre Peteraf (1993), permet à des firmes qui n'ont pas les meilleures ressources de rester sur le marché si leurs inputs, hétérogènes, ont la productivité minimale. Puisque les ressources qui permettent d'obtenir des rentes sont en quantités limitées, des firmes avec une efficacité productive (capacité) moindre peuvent rester sur le marché afin de satisfaire toute la demande. Peteraf (1993) donne une illustration de ce cas en faisant l'hypothèse que la firme avec les meilleures ressources a un coût moyen plus faible.

Marshall introduit également la notion de « quasi-rente composite ». Lorsque la rente de deux actifs employés ensemble est supérieure à la rente des actifs pris séparément, alors cette rente est appelée « quasi-rente composite ». Marshall (1920:626) donne l'exemple d'un moulin à eau et d'une usine d'énergie hydraulique. On peut supposer que les deux ressources ont été conçues de façon à être co-spécialisées par des investissements spécifiques. Ces investissements créent une dépendance bilatérale dans l'utilisation des ressources, Teece (1980) décrit cette notion de co-spécialisation. Amit et Schoemaker (1993) emploient la notion de complémentarité. Dierickx et Cool (1989) parlent d'interconnexion. 
Les rentes Ricardo - Marshaliennes se distinguent des rentes de monopole comme le note Peteraf (1993:191) par le fait que, pour ces dernières, l'offre est délibérément limitée par rapport aux ressources disponibles. Il s'agit dans le cas des rentes de monopole d'une pénurie organisée du bien, soit par les décisions et les efforts de la firme, soit d'une façon institutionnalisée par la mise en place de droits de propriété comme les brevets ou de l'obtention d'une exclusivité d'exploitation d'une mine.

Ce concept de rente basé sur la structure de la concurrence peut être complété avec le concept de rente à la Chamberlin (1933). Pour Teece et al.(1997) la recherche de ce type de rente est l'objectif le plus communément rencontré dans le champ théorique de l'économie industrielle. Dans ce modèle l'offre d'un bien est faite par une seule entreprise, comme dans le cas du monopole, mais il est tenu compte de l'existence de substituts de ce bien qui lui font concurrence. Cependant l'existence d'un produit de substitution n'implique pas que ce produit est offert au même prix ou à un prix inférieur, ni qu'il soit un substitut dans toutes les dimensions du produit. Une rente à la Chamberlin est donc une rente de monopole que la firme obtient parce qu'elle possède un avantage dans une dimension particulière de ce bien, qui, compte tenu de la structure de la concurrence, ne peut être éliminé par le jeu de marché.

Si la rente à la Ricardo-Marshall comme on vient de le présenter provient d'un stock de ressources accumulées, cette accumulation est le fait de décisions prises par la firme (décision de constituer un stock de capital physique et humain Dierickx et Cool (1989) pour organiser une pénurie délibérée). L'existence de rentes de monopole - Chamberlin, quant à elles, correspondent à la fois à des compétences dynamiques propres à la firme (comme la possibilité de limiter le débit des ressources) mais également à l'inexistence de compétences dynamiques des firmes concurrentes qui ne sont pas en mesure d'imiter ou de substituer les ressources considérées. Enfin, si certaines ressources doivent être combinées pour créer des rentes, ce processus de création est alors nécessairement transversal à la firme et ne peut se limiter à certaines unités.

La détermination de la quantité optimale de ressources à développer s'interprète comme un calcul d'option et non d'une simple optimisation. En effet, une simple optimisation ne prendrait pas en compte les gains possibles provenant des combinaisons de ressources inattendues mais uniquement de leur emploi défini dans le programme d'optimisation. Le gain en provenance d'autres combinaisons correspond aux applications des options de croissance comme décrite par (Kester, 1984), notamment le développement de la firme multi-produit. Les options emploient également une référence temporelle quant au moment où les ressources doivent être développées et mises en service (Madj and Pindyck, 1987). Cette littérature se base surtout sur des investissements en capitaux physiques (machines), or les rentes peuvent parfaitement venir d'un stock de ressources intangibles, ce point est développé ci-après.

\section{III.2. Rentes d'informations et de connaissances}

Milgrom et Roberts (1992) définissent les rentes d'informations comme les gains supérieurs d'un individu qui détient des informations privées avant la signature d'un contrat. Afin d'éviter l'opportunisme pré-contractuel d'une partie (comme celle rencontrée dans la sélection adverse), cette partie doit recevoir des incitations pour ne pas tirer profit de ses informations privées. Ces incitations constituent une rente. Rebérioux (2003) propose une approche de la gouvernance où la répartition de ce type 
de rente est étudiée sur la base d'une séparation des fonctions de direction et de contrôle.

L'approche de Milgrom et Roberts prend place dans le cadre de l'économie de l'information où des éléments comme la confiance et la réputation jouent un rôle majeur. La confiance mutuelle que deux parties peuvent avoir lorsqu'elles s'engagent dans un contrat se retrouve dans le concept de rentes relationnelles, Dyer et Singh (1998 :662). Shapiro (1983) donne un exemple où la ressource intangible est représentée par la réputation. Le flux de rente est corrélé à la réputation, ce qui constitue une incitation pour la construire et la maintenir.

La prise en compte de l'influence de l'information des différentes parties sur les rentes conduit Schoemaker (1990) à s'interroger sur la rationalité associée à la création de rentes. La création d'une rente, pour cet auteur, est possible suivant les niveaux de rationalité et d'efficience des marchés retenus. Aussi, plus la rationalité est forte plus les rentes seront des quasi-rentes de très courte période. Dans le cas opposé, avec des marchés imparfaits et une rationalité limitée il est possible de créer de façon délibérée une rente de long terme. Cohendet et Llerena (1990) montrent le rôle de l'information dans l'évaluation d'une firme et l'importance du passage de la rationalité absolue à une rationalité procédurale.

Une approche de la firme basée sur les connaissances et non l'information (Cohendet et Llerena, 1999) modifie la nature des rentes, leur création et leur appropriation. Selon Teece (1993) le rôle fondamental du manager est de gouverner la firme de telle façon à générer, augmenter, et protéger ce type de rentes.

La création de rentes basées sur la connaissance sont analysées notamment par Spender (1996). Pour cet auteur différent types de connaissances conduisent à des rentes de natures différentes. Ainsi la connaissance collective conduit à des rentes dites à la Penrose tandis que des connaissances explicites donnent lieu à des quasi-rentes de monopole découlant de ces connaissances. La connaissance collective comprend à la fois le « meaning » regroupant l'aspect cognitif, affectif, symbolique et culturel lié à la connaissance et le " praxis » regroupant l'aspect comportemental, rituel et les routines organisationnelles. Cette connaissance collective est par nature ancrée à la firme d'une façon historique, relativement immobile, spécifique à la firme. Elle est donc relativement inimitable. Par contre les connaissances explicites, conscientes et objectives induisent des quasi-rentes de monopole qui peuvent être dissipées par une firme concurrente qui imite la connaissance sous-jacente, Spender(1996:73). L'appropriation de la rente devient alors fondamentale, Klein et al.(1978).

La prise en compte du temps dans les changements de rentes se fait notamment par la notion de coûts de transactions dynamiques. Il s'agit des coûts subis par la firme lorsque celle-ci n'a pas la capacité de production qu'elle nécessite à un instant donné. Langlois (1992) remarque que les ressources n'ont pas le même coût d'acquisition pour toutes les firmes, celui-ci dépend du stock de ressources existants et de l'ordre d'accumulation, aussi certaines firmes accordent une valeur plus élevés a des ressources que d'autres. Ces coûts de transactions sont dus à une asymétrie d'information lors de l'acquisition de ressources Les coûts de transactions dynamiques font le lien entre les rentes basées sur de l'information et celles provenant de connaissances. Aussi l'espérance de rente ne doit 
pas être la même pour toute les firmes, puisqu'une firme dont les coûts de transaction dynamiques sont élevés doit formuler des anticipations de rentes plus élevés.

\section{III.3. Rentes entrepreneuriales, d'innovation, à la Schumpeter}

Les concepts de rentes précédents s'appliquent à une industrie stable. Ils comparent la productivité de ressources dans différentes utilisations dont la valeur est connue ex ante, ou suffisamment connue pour être appréciée en terme de risque. La rente entrepreneuriale par contraste est la découverte de nouvelles combinaisons de ressources dont l'incertitude est une caractéristique fondamentale (rentes entrepreneuriales, d'innovation et à la Schumpeter sont synonymes dans la littérature). Rumelt (1987) définit la rente entrepreneuriale comme la différence entre la valeur ex post d'un projet (ou du flux de revenu qu'il génère) et le coût ex ante des ressources combinées pour réaliser ce projet. Si on pose l'hypothèse d'un équilibre anticipé (coût ex ante $=$ valeur ex post) alors la rente entrepreneuriale est nulle.

Cette définition implique que la rente entrepreneuriale est nécessairement temporaire. Lorsque les visions des autres acteurs de l'industrie sont les mêmes que les anticipations de l'entrepreneur, cette forme de rente sera amenée à évoluer. Ainsi une firme qui a développé une innovation dont l'impact ne sera apprécié correctement qu'un certain temps après sa mise sur le marché aura une quasi-rente, voire une rente de monopole en fonction du délai nécessaire aux firmes concurrentes pour acquérir les connaissances nécessaires pour produire un tel bien et la concurrencer.

Phelan et Lewin (2002) décrivent ce changement en terme d'équilibre et déséquilibre de marché selon le mode de pensée de l'école Autrichienne. Ces auteurs notent que toutes les rentes peuvent trouver leur origine dans un état de déséquilibre généré par une innovation et sont en substance une rente à la Schumpeter. Par contre la rente entrepreneuriale n'a pas d'homologue dans une situation d'équilibre (l'opportunisme ne conduisant pas à une rente, mais à un profit qui s'estompe par le jeu de la concurrence).

Rumelt (1987), qui a introduit le concept de rente entrepreneuriale, distingue essentiellement trois caractéristiques qui forment l'originalité de ce type de rente:

(i) L'incertitude joue un grand rôle dans la production de rentes entrepreneuriales.

En absence d'incertitude on peut s'attendre à ce que les inputs utilisés reflètent leur vraie valeur. Leur utilisation donne lieu à une imitation rapide afin de réduire le profit à un niveau normal. L'incertitude sur les possibilités de production ex ante bloque l'imitation.

(ii) L'innovation entrepreneuriale pour produire une rente doit être «socialement efficace». C'est à dire que l'innovation doit conduire à une augmentation de valeur suffisamment forte par rapport aux substituts préexistants pour justifier les coûts de l'innovation. Il s'agit là d'une condition d'appartenance à la catégorie innovation et non pas d'invention au sens de Schumpeter.

(iii) L'existence de la rente dépend des caractéristiques de l'entrepreneur qui arrive à rompre les freins à la création comme l'ambiguïté causale (Lippman et Rumelt, 1982). 


\section{IV. - CONCLUSION}

La multitude de rentes existantes rend difficile leurs analyse, en particulier dans un modèle d'économie industrielle, malgré les efforts de modélisation (Lippman et Rumelt, 2003), l'approche la plus claire actuellement est de type conceptuel, c'est ce que l'on propose dans la suite.

On peut, à la lumière des réflexions précédentes, dire que les compétences dynamiques sont les processus qui font évoluer la firme d'une façon décidée dans le principe. Ces processus proviennent et utilisent des ressources pour reconfigurer, créer et détruire des ressources, afin de faire face à des changements dans l'industrie. Les compétences dynamiques sont les routines organisationnelles et stratégiques grâce auxquelles la firme arrive à obtenir une nouvelle configuration des ressources lorsque des marchés émergent, évoluent et disparaissent. La configuration de ressources obtenues permet de créer et de capturer des rentes spécifiques en fonction du dynamisme de l'industrie. Le point suivant présente un lien entre rentes et capacités dynamiques en guise de conclusion.

\section{IV.1. Les relations rentes capacités dynamiques}

La figure 2 est une représentation des mécanismes qui lient les différentes rentes aux compétences dynamiques, et in fine, aux processus à l'œuvre pour obtenir des capacités dynamiques. Sur ce graphique les catégories de rentes que la firme peut obtenir sont réparties dans les trois catégories que l'on a présenté : les rentres de monopole ou de Chamberlin obtenu via un output que la firme est seule à pouvoir produire dans des conditions d'efficience particulières, les rentes provenant des stocks d'inputs sans lien direct avec un output précis, enfin les rentes à la Schumpeter. La revue de la notion de rente montre que chaque rente est à l'origine une rente entrepreneuriale, reprenant les catégories de Schumpeter (1935), la fabrication d'un bien nouveau, la mise en place d'une nouvelle méthode de production, l'ouverture d'un débouché nouveau, la conquête d'une nouvelle source d'inputs, ou la mise en place d'une nouvelle organisation.

Insérer ici Figure 2

Selon le type de firmes dans l'industrie, l'environnement concurrentiel sera stable ou dynamique et conduira à transformer la rente à la Schumpeter en rente provenant d'un input ou d'un output. Un faible environnement concurrentiel implique que les firmes n'arrivent pas à imiter avec succès la firme innovatrice qui recueille une rentre entrepreneuriale. Cette dernière se place alors dans une situation de monopole. A l'inverse si les firmes concurrentes ont des compétences dynamiques qui leurs permettent d'avoir une réaction au moins $a d$ hoc, et de réagir sur certaines dimensions aux nouvelles compétences de la firme innovatrice alors les firmes sont capables de produire sans avoir le niveau d'efficience (la capacité) de la firme innovatrice. On retrouve ainsi le cas décrit par Peteraf (1993) une firme à la capacité de production plus efficace, mais, pas suffisamment pour éradiquer toute concurrence qui subsiste et sert une partie de la demande. L'industrie est dans ce cas composée de firmes hétérogènes, les meilleures perçoivent des rentes grâce à leurs ressources (au sens large du terme incorporant les actifs tangibles et intangibles). 


\section{IV.2. Discussion}

Ce travail tisse des liens entre les notions polymorphes de capacités dynamiques et de rentes. Cette discussion prend place dans un débat plus vaste sur les interactions entre les champs du management stratégique et l'économie industrielle. Si le premier s'oriente vers la problématique des connaissances et de l'apprentissage comme source de rente, le second développe massivement la problématique de la structure industrielle et de la position dominante pour produire des rentes.

Si ces deux approches peuvent se conjuguer, elles n'apportent pour l'instant que peu d'éléments pour guider le choix des ressources à développer. Le processus de décision esquissé par Winter est limité à la problématique évolutionniste. La firme est liée d'une façon durable et coûteuse à ses ressources, il en résulte une perte de flexibilité, Cohendet et Llerena (1989). Aussi faut-il guider le choix des ressources via des critères financiers, économiques (économie industrielle, coûts de transaction) tout en prenant en compte la particularité des ressources intangibles comme la connaissance.

Aussi ne s'agit-il pas de choisir simplement parmi un menu offert à la firme, mais de créer et de développer ce menu de choix. Cette optique, comme le suggère Dosi et al.(2000), inclue la dynamique d'apprentissage et nécessite probablement le développement de nouveaux outils.

Ces outils devraient notamment (i) prendre compte de la dépendance de sentiers des investissements tangibles et intangibles et des choix futurs qu'ils autorisent. Puisque la position d'une firme à un instant donné, exprimé en terme de capacité dynamique dépend de ces compétences et ressources accumulées, il est clair que ses choix d'évolution futurs sont dépendants de son évolution passé. (ii) Spécifier le rôle de l'intentionnalité et des agents qui exercent les décisions. Il s'agit, comme l'a noté Selznick, que la firme dans son hétérogénéité forme un tissu social vivant avec, pour être efficace un ou des organes de décisions et une vision qui transcendent la compréhension purement technique de son fonctionnement. (iii) Enfin, prendre en compte la multiplicité des processus co-évoluant sein de la firme qui influence aussi bien les coûts que les seuils de satisfaction et de motivation des agents.

Un outil répondant, au moins partiellement, à ces besoins semble être les options réelles. Si ce champ théorique connaît de nombreux développement en économie industrielle et en management stratégique, il n'y pas, pour l'instant, de travaux l'intégrant dans une théorie de la firme prenant explicitement en considération l'économie de la connaissance. Il semble cependant très prometteur comme le suggère Denrell et al.(2003) et Winter (2000:983) «An organizational capability (...) together with its implementing inputs flows, confers upon an organization's management a set of decision options for producing significant outputs of a particular type » 


\section{Bibliographie}

ALCHIAN, A.A., (1988), "Rent ", The New Palgrave - a dictionary of economics, MacMillan, London, UK.

AMIT, R. et SCHOEMAKER, P.J.H, (1993), "Strategic assets and organizational rent », Strategic Management Journal, vol.4, n²1, pp. 33-46.

AZOULAY, N. et WEINSTEIN, O., (2000), "Les compétences de la firme", Revue d'Économie Industrielle, n 93, 4ème trim., pp.117-154.

BARNEY, J., (1991), «Firm Resources and Sustained Competitive Advantage », Journal of Management, vol.17, $\mathrm{n}^{\circ} 1$, pp.99-120.

CHAMBERLIN, E., (1933), The Theory of Monopolistic Competition, 8th ed., Cambridge, Harvard University Press.

COHENDET, P. et LLERENA, P., (1989), Flexibilité, Information et Décision, Economica, Paris.

COHENDET, P. et LLERENA, P., (1990), "Nature de l'information, évaluation et organisation de l'entreprise », Revue d'Économie Industrielle, n51, 1 ${ }^{\mathrm{er}}$ trim., pp.141-165.

COHENDET, P. et LLERENA, P., (1999), « La conception de la firme comme processeur de connaissances », Revue d'Économie Industrielle, n88, 2ème trim., pp.211-236.

COLLIS, D.J., (1994), "How Valuable Are Organizational Capabilities ? ", Strategic Management Journal, Winter, vol.15, pp.143-152.

DENRELL, J. et FANG, C. et WINTER, S.G., (2003), " The Economics of Strategic Opportunity », Strategic Management Journal, vol.24, pp.977-990.

DE TONI, A. et TONCHIA, S., (2005), « Definitions and linkages between operational and strategic flexibilities », Omega, forthcoming.

DIERICKX, I. et COOL, K., (1989), " Asset Stock Accumulation and Sustainability of Competitive Advantage », Management Science, vol.35, n²12, pp.1504-1511.

DOSI, G. et NELSON, R.R. et WINTER, S.G., (2000), "Introduction : The Nature and Dynamics of Organizational Capabilities », in: Dosi, G. et Nelson, R.R. et Winter, S.G (ed.), Nature \& Dynamics of Organizational Capabilities, Oxford University Press, New York.

DYER, J.H. et SINGH, H., (1998), « The relational view: cooperative strategy and sources of interorganizational competitive advantage », Academy of Management Review, vol.23, $\mathrm{n}^{\circ} 4, \mathrm{pp} .660-679$.

EISENHARDT, K. et MARTIN, J., (2000), " Dynamic capabilities: What are they ? ", Strategic Management Journal, vol.21, pp.1105-1121. 
FOSS, N.J., (1996), "Capabilities and the theory of the firm », Revue d'Économie Industrielle, vol.77, 3ème trim., pp.7-28.

FOSS, N.J., (1997), Resources and Strategy: A Reader, Oxford: Oxford University Press.

HAYES, R.H. et PISANO, G.P. et UPTON, D.M. et WHEELWRIGHT, S.C., (2004), Operations, Strategy, and Technology: Pursuing the Competitive Edge, John Wiley \& Sons, New-York.

HODGSON, G.M., (1998), "Evolutionary and competence-based theories of the firm », Journal of Economic Studies, vol.25, n¹, pp.25-56.

KESTER, W.C., (1984), « Today's options for tomorrow's growth », Harvard Business Review, vol. 62, pp.153-160.

KLEIN, B. et CRAWFORD, R.G. et ALCHIAN, A.A., (1978), "Vertical Integration, Appropriable Rents, and the Competitive Contracting Process », Journal of Law and Economics, vol.21, n², pp.297-326.

KOGUT, B. et ZANDER, U., (1992), « Knowledge of the firm, combinative capabilities, and the replication of technology », Organisation Science, vol.3, pp.383-397.

LANGLOIS, R.N., (1992), "Transaction-cost Economics in Real Time », Industrial and Corporate Change, vol.1, $\mathrm{n}^{\circ} 1$, pp.99-127.

LEWIN, P. et PHELAN, S.E., (2002), « Rent and resources : a market process perspective », in: Foss, N.J. et Klein, P.G. (ed.), Entrepreneurship and the Firm, Austrian Perspectives on economic organization, Edward Elgar, Cheltenham, UK.

LIPPMAN, S.A. et RUMELT, R.P., (1982), « Uncertain imitability: an analysis of interfirm differences in efficiency under competition », Bell Journal of Economics, vol.13, pp.418438.

LIPPMAN, S.A. et RUMELT, R.P., (2003), « The Payments Perspective: Micro-Foundations of Resource Analysis », Strategic Management Journal, vol.24, pp.903-927.

LLERENA, P. et OLTRA, V., (2002), « Diversité des processus d'apprentissage et efficacité dynamiques des structures industrielles ", Revue d'Économie Industrielle, vol.98, $1^{\mathrm{er}}$ trim., pp.95-120.

MADJ, S. et PINDYCK, R., (1987), " Time to build, option value, and investment decisions», Journal of Financial Economics, vol.18, pp.7-27.

MAKADOK, R., (2001), « Toward a Synthesis of resource-based and Dynamic-Capability Views of Rent Creation », Strategic Management Journal, vol.22, pp.387-401.

MILGROM, P. et ROBERTS, J., (1992), Economics, Organisation and Management, Prentice-Hall, New Jersey. 
MUTH, R.F., (1968), « Rent », International Encyclopaedia of the Social Science, MacMillan and FreePress.

PENROSE, E.T., (1959), The Theory of the Growth of the firm, Oxford University Press.

PETERAF, M.A., (1993), « The cornerstones of competitive advantage: A resource-based view », Strategic Management Journal, vol.14, pp.179-191.

PORTER, M.E, (1991), « Towards a dynamic theory of strategy », Strategic Management Journal, vol.18, 5, pp.95-117.

PORTER, M.E., (1996), "What is Strategy ? ", Harvard Business Review, NovemberDecember, pp.61-78.

RATHE, K. et WITT, U., (2001), " The nature of the firm - Static versus developmental interpretation », Journal of Management and Governance, vol.5, pp.331-351.

REBERIOUX, A., (2003), «Gouvernance d'entreprise et théorie de la firme : quelle(s) alternative(s) à la valeur actionnariale?», Revue d'Economie Industrielle, vol.104, 4ème trim., pp.85-110.

RICARDO, D., (1821), The Principles of Political Economy and Taxation, London, The Guernsey Press.

RUMELT, R.P., (1987), " Theory, Strategy and entrepreneurship », in: Teece, D.J. (ed.), The Competitive Challenge, Strategies for Industrial Innovation and Renewal, Ballinger Publishing Company, Cambridge, Massachusetts.

SCHOEMAKER, P.J.H., (1990), "Strategy, Complexity and Economic Rent », Management Science, vol.36, $\mathrm{n}^{\circ} 10$, pp.1178-1192.

SCHUMPETER, J.A., (1935), Théorie de l'évolution économique, deuxième édition, Librairie Dalloz, Paris.

SELZNICK, P., (1957), Leadership in Administration: A Sociological Interpretation, Row, Peterson, and Co., Evanston, IL.

SHAPIRO, C., (1983), " Premiums for high quality products as rent to reputation », Quarterly Journal of Economics, vol.98, pp.659-680.

SPANOS, Y.E. et LIOUKAS, S., (2001), " An examination into the causal logic of rent generation: Contrasting Porter's competitive strategy framework and the resourcebased perspective », Strategic Management Journal, vol.22, pp.907-934.

SPENDER, J.C., (1996), " Making Knowledge the Basis of a Dynamic Theory of the Firm », Strategic Management Journal, vol.17, pp.45-62. 
STANKIEWICZ, F., (2003), « Des compétences de la firme aux compétences des salariés : le point de vue non autorisé d'un économiste du travail », Revue d'Économie Industrielle, vol.102, $1^{\text {er }}$ trim., pp.55-68.

TEECE, D.J., (1980), « Economies of Scope and the Scope of the Enterprise », Journal of Economic Behaviour and Organization, vol.1, pp.223-247.

TEECE, D.J., (1993), « The Dynamics of Industrial Capitalism: Perspectives on Alfred Chandler's Scale and Scope », Journal of Economic Literature, vol.31, pp.199-225.

TEECE, D. J. et PISANO, A., (1994), « The Dynamic Capabilities of Firms : An Introduction », Industrial and Corporate Change, vol.3, n³, pp.537-556.

TEECE, D.J. et PISANO, G. et SHUEN, A., (1997). « Dynamic capabilities and strategic management », Strategic Management Journal, vol.18, pp.509-533.

WERNERFELT, B., (1984), «A Resource-based View of the Firm », Strategic Management Journal, vol.5, n², pp.171-180.

WINTER, S.G., (2000), " The Satisficing Principle in Capability Learning », Strategic Management Journal, vol.21, pp.981-996.

WINTER, S.G., (2003), «Understanding Dynamic Capabilities », Strategic Management Journal, vol.24, pp.991-995.

ZOLLO, M. et Winter, S.G., (2002), « Deliberate Learning and the Evolution of Dynamic Capabilities », Organization Science, vol.13, n³, pp.339-351. 


\title{
Tableau 1 : Les principales définitions des compétences dynamiques
}

\begin{abstract}
" By combinative capabilities, we mean the intersection of the capability of the firm to exploit its knowledge and the unexplored potential of the technology.", Kogut et Zander (1992:391).

" The term 'dynamic' refers to the shifting character of the environment; certain strategic responses are required when time-to-market and timing is critical, the pace of innovation is accelerating, and the nature of future competition and markets is difficult to determine. The term 'capabilities' emphasizes the key role of strategic management in appropriately adapting, integrating, and re-configuring internal and external organizational skills, resources, and functional competences toward changing environment.", Teece et
\end{abstract} Pisano (1994:538).

"We refer to this ability to achieve new forms of competitive advantage as 'dynamic capabilities' (...) The term 'dynamic' refers to the capacity to renew competences so as to achieve congruence with the changing business environment (...) The term 'capabilities' emphasizes the key role of strategic management in appropriately adapting, integrating and reconfiguring internal and external organizational skills, resources and functional competences to match the requirements of an changing environment." "We define dynamic capabilities as the firms ability to integrate, build, and reconfigure internal and external competences to address rapidly changing environments. Dynamic capabilities thus reflect an organization's ability to achieve new and innovative forms of competitive advantage given path dependencies and market positions.", Teece, Pisano et Shuen (1997:515-516).

“The firm's processes that use resources -specifically the processes to integrate, reconfigure, gain and release resources-to match and even create market change. Dynamic capabilities thus are the organizational and strategic routines by which firms achieve new resource configurations as markets emerge, collide, split, evolve, and die.", Eisenhardt et Martin (2000:1107).

"A dynamic capability is a learned and stable pattern of collective activity through which the organization systematically generates and modifies its operating routines in pursuit of improved effectiveness.", Zollo et Winter (2002:340).

"An organizational capability is a high level routine (or collective routines) that together with its implementing inputs flows, confers upon an organization's management a set of decision options for producing significant outputs of a particular type.", Winter (2003:991), Winter (2000:983). 
Figure 1: Options et dynamique de production

(adaptée de Hayes et al., 2004 ; De Toni et Tonchia, 2005)
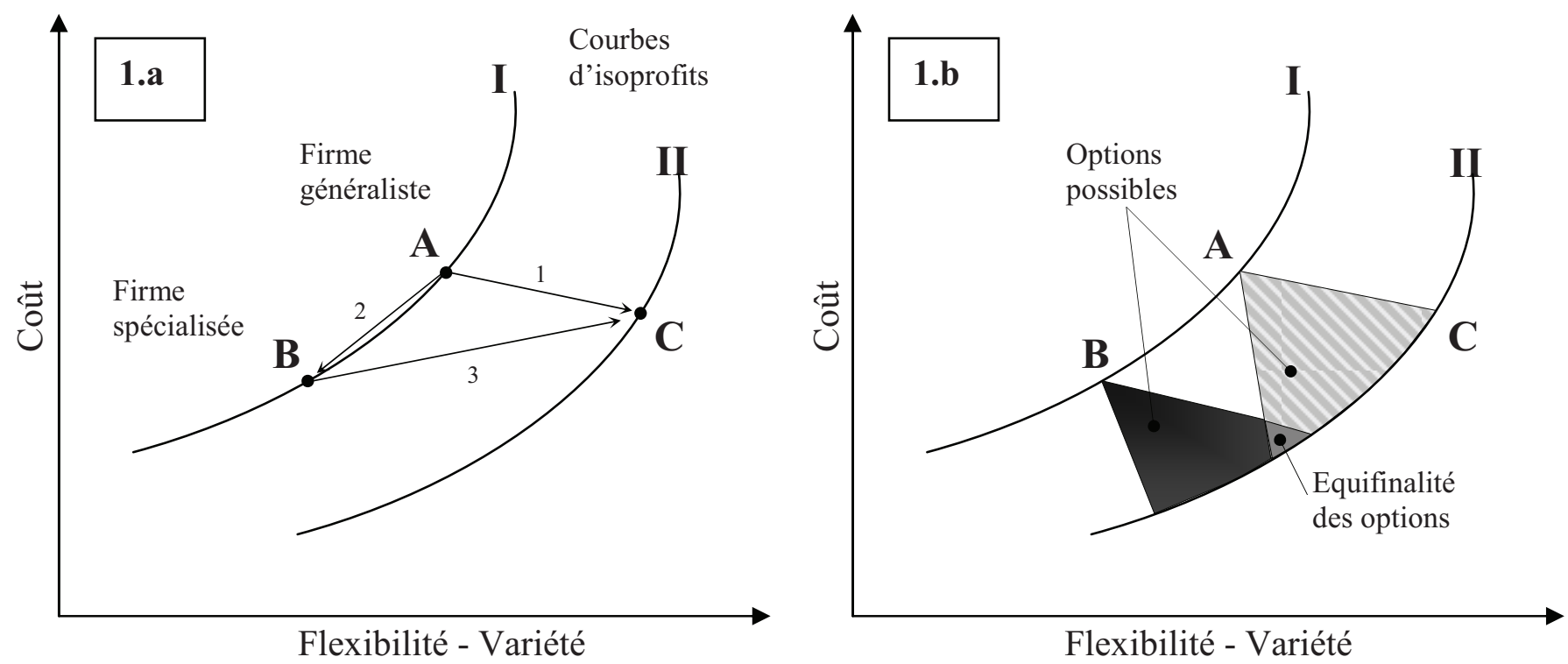
Figure 2: Relations rentes - capacités dynamiques

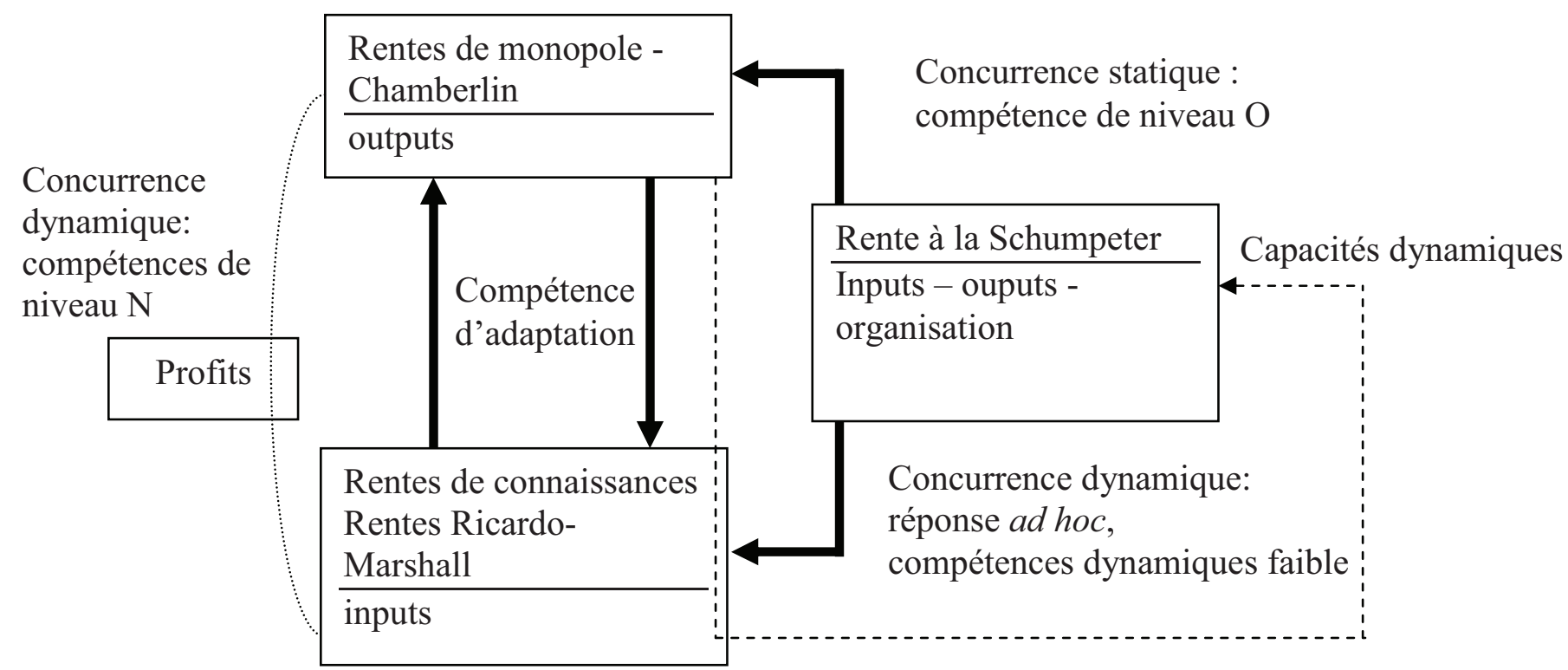




\section{Documents de travail du BETA}

2011-01 La création de rentes : une approche par les compétences et capacités dynamiques Thierry BURGER-HELMCHEN, Laurence FRANK, janvier 2011.

La présente liste ne comprend que les Documents de Travail publiés à partir du $1^{\mathrm{er}}$ janvier 2011. La liste complète peut être donnée sur demande.

This list contains the Working Paper writen after January 2011, 1rst. The complet list is available upon request. 